\title{
THE METHOD OF AUTOMATIC IDENTIFICATION OF MOTOR VEHICLE USERS
}

\author{
Tadeusz Niedziela ${ }^{1}$ (D) \\ ${ }^{1}$ University of Technology and Humanities, Faculty of Transport, Electrical Engineering and Computer Science, Malczewskiego 29, \\ 26-600 Radom, Poland, t.niedziela@uthrad.pl, https://orcid.org/0000-0002-4772-3797
}

Reviewed positively: 19.05 .2020

Information about quoting an article:

Niedziela T. (2020). The method of automatic identification of motor vehicle users. Journal of civil engineering and transport. 2(2), 63-71, ISSN 2658-1698, e-ISSN 2658-2120, DOI: 10.24136/tren.2020.005

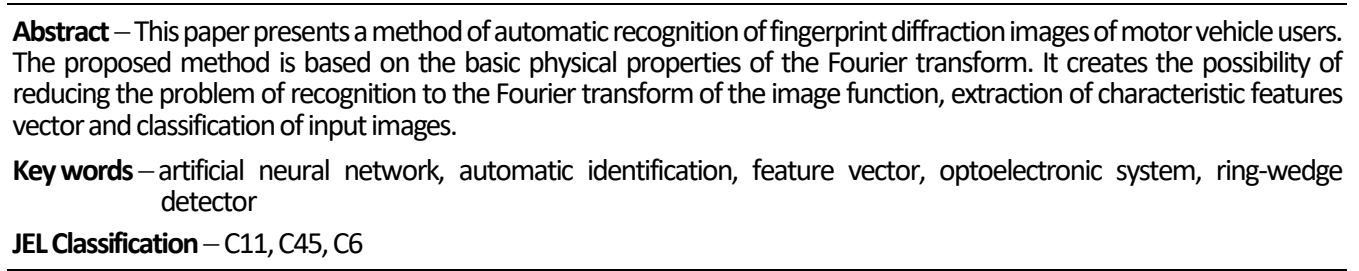

\section{INTRODUCTION}

The Automatic Target Recognition from images (ATR) is one of the major contemporary research problems [1-11]. Many researchers and companies are trying to build a system that is both reliable, fast and easily adaptable. Hybrid (optoelectronic) solutions play a significant role among these systems. They are designed mainly for large parallel calculations, without significant delays. The final processing of optical results is most often done in a computer, often using artificial intelligence methods (artificial neural network - ANN).

Classic automatic recognition algorithms consist of two steps: vector's extraction of the characteristic features of objects from images, in which there is most often a high level of noise, and classification or identification. Hybrid (optoelectronic) systems play an important role among such systems.

Characteristic features of the object can be extracted directly from the image or from its transformation. The type of image determines whether features are detected from the image or its transform. Images with a complex internal structure (texture) are easier to analyze in the space of their transforms, while images with a poor structure are more convenient to describe using characteristics extracted directly from the image.
The construction of the feature vector allows for a significant reduction in the amount of information to be further processed and speeds up the recognition process.

The identity or similarity of the recognized objects can be inferred based on the difference of characteristic features' vectors, both in the image space and in the space of their transform.

The purpose of this work is to show the current possibilities in automating the identification of motor vehicle users.

\section{OPTOELECTRONIC SYSTEM OF AUTOMATIC RECOGNITION IN THE DIFFRACTION SPACE}

The proposed automatic recognition system (Fig. 1) allows the characteristic features' extraction of the invariant objects relative to scale, rotation and displacement. The basis of the system is an extractor of characteristic features in the form of a ring-wedge detector RWD and a classifier in the form of an artificial neural network ANN. The main advantage of this solution is the relatively low cost and possibility of computer simulations.

The basic idea is the practical use of the Fourier transform properties implemented by optical means. Optical methods for implementing this transform are simple and are currently finding wider and wider applications. 


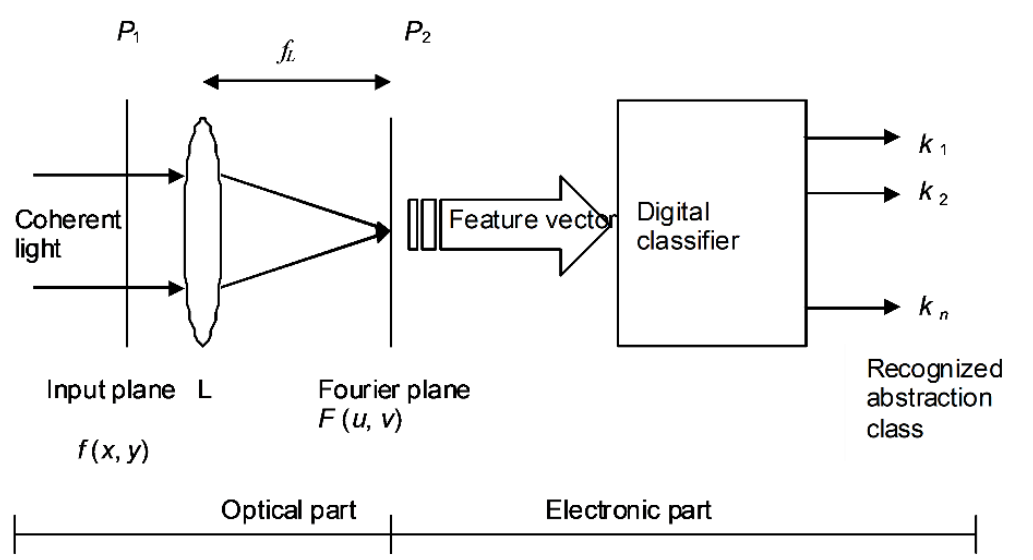

Fig. 1. Schematic diagram of the optoelectronic (hybrid) image recognition system in the diffraction plane

a)

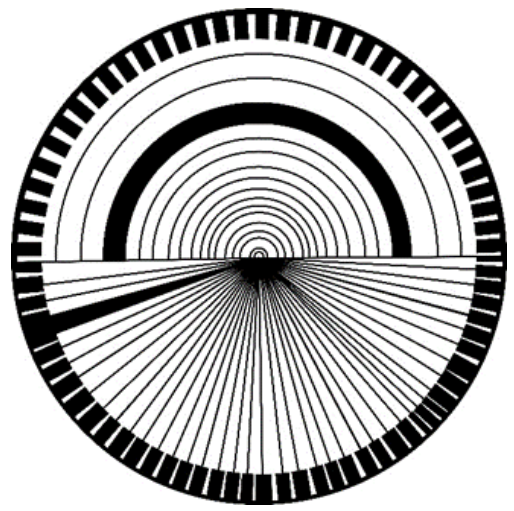

b)

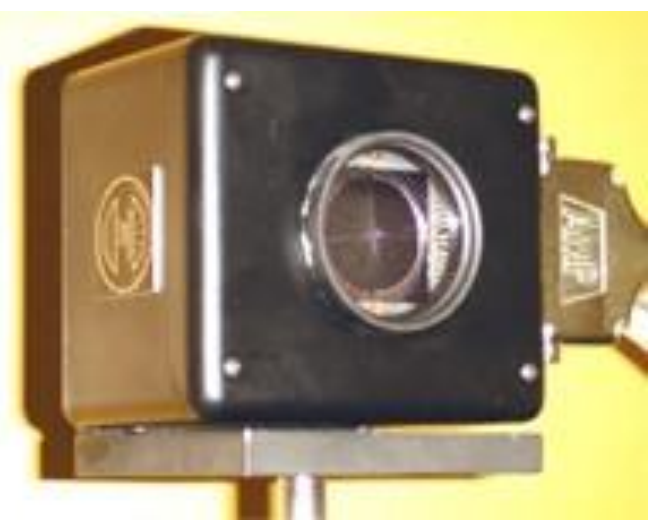

Fig. 2. Schematic diagram of the extractor of characteristic features in the form of RWD matrix; a) schematic diagram, b) laboratory model

\section{THE EXTRACTOR OF CHARACTERISTIC FEATURES} IN THE FORM OF A RING-WEDGE DETECTOR

The complete information about the Fourier spectrum of the lens is contained in its one halfplane. Thus, the focal plane of the lens can be divided into two half-planes and each of them can be sampled separately with the help of specialized detectors in the form of wedges and rings (Fig. 2). Each of the detector elements integrates the image of the spectrum incident on the surface resulting in

a strictly defined numerical value - exactly one features' vector.
The Fraunhofer approximation of the Fresnel-Kirchhoff integral realizes by lens can be

expressed as [6] (1):

$$
F(\xi, \eta, z)=\frac{i E_{0}}{\lambda z} e^{-\frac{i \pi}{\lambda z}\left(\xi^{2}+\eta^{2}\right)} \int_{-\infty}^{\infty} \int_{-\infty}^{\infty} f(x, y) e^{\frac{i 2 \pi}{\lambda z}\left(x \xi^{2}+y \eta^{2}\right)} d x d y
$$

characteristic feature of the image. It is possible to filter selected components of the image spectrum, e.g. by obscuring selected sectors on the detection plane.

If the extractor of characteristic features in the form of a ring-wedge detectors' matrix is placed in the rear focal plane of the lens (in the Fourier plane), then it is possible of sampling diffraction images - sampling spectral power, which is focused on rings and wedges. Thanks to this, each area of RWD generates one element of the characteristic features' vector. 
which in practice gives the Fourier transform of the input function $f(x, y)$ with the result multiplied by the spherical phase parameter.

Since only the intensity can be directly observed and recorded in practical applications, the spherical phase parameter can be omitted. Therefore the intensity is the spectral power of Fourier transmittance $F^{2}(u, v)$ of the input function $f(x, y)$, which is called the Fraunhofer diffraction pattern.

If the Fraunhofer diffraction pattern is expressed in polar coordinates $(\omega, \varphi)$, then the characteristic features of the object registered by the rings $R_{i}$ and the features recorded by the wedges $W_{i}$ can be expressed as (2):

$$
\begin{aligned}
& R_{i}=\int_{0}^{\pi}\left[\int_{r_{i}}^{r_{i+1}} F^{2}(\omega, \varphi) d \omega\right] d \varphi \\
& W_{i}=\int_{0}^{R}\left[\int_{\varphi_{i}}^{\varphi_{i+1}} F^{2}(\omega, \varphi) d \varphi\right] d \omega
\end{aligned}
$$

where: $r_{i}$ and $r_{i+1}$ are the inner and outer radius of the ring, respectively (RING (i)), $\varphi_{i}$ and $\varphi_{i+1}$ are mean the start angle and the end angle of the wedge, respectively (WEDGE (i), $\varphi_{j}\left(j=1, \ldots, N_{w}\right)$, while $\mathrm{R}$ is the radius of ring-wedge detector RWD.

Therefore, the values of the extracted characteristic features $\mathrm{R}$ through the wedges are invariant with respect to the shift and rotation of the input image. On the other hand, the values of

the characteristic features $W$ extracted by the halfrings are invariant with respect to the shift and change of input image scale.

What invariances in the feature vector are important, in practice is a problem in itself.

\section{CLASSIFIER IN THE FORM OF ANN}

Noise in images is usually the sum of elementary noises caused by many independent phenomena and has a Gaussian distribution, hence the classification should be based on the statistical uncertainty model. In this model, the predicted conditional probability $\mathrm{P}\left(\mathrm{C}_{\mathrm{k}} \mid \mathrm{x}\right)$ (a'posteriori) is used as a criterion for the decision to minimize average losses caused by incorrect classification. Classification is a mapping of $\delta(x)$, from the space of characteristic features' vectors $X$ to the space of sets of classes $C$. For supervised classification, the mapping is in the form of $P$ examples $\left(X^{i}, C_{j(i)}\right)$, where: $X^{i}(i=1, \ldots, P)$ are characteristic features vectors, and $C_{j(i)}(j(i)=1, \ldots, M)$ are classes related to feature vectors, respectively. The optimal classifier in the probabilistic uncertainty model is one that minimizes Bayes risk $R$ understood as [6] (3):

$$
R=\sum_{i=1}^{M} \sum_{j=1}^{M} L_{i j} P_{j} \int \ldots \int_{v \in D_{i}} P\left(X \mid C_{j}\right) d x
$$

where: $P_{j}$ is the probability a'priori of the class $C_{j}$, $D_{i}$ is the area in the space of characteristic features in which each point is assigned to the class $C_{i}$ and $L_{i}$ is at the expense of the decision: $C_{i}$ when $C_{j}$ is true.

Assuming that $L_{i j}=1-d_{i j}$, where $d_{i j}$ is a Kronecker symbol, and by developing equation ( 3 ) for $j=i$, and $I \neq j$ and Bayes risk is got in the form (4):

$$
R=\sum_{i=1}^{M} \sum_{j \neq 1} P_{j} \int \ldots \int_{x \in D_{i}} P\left(X \mid C_{j}\right) d x=\sum_{i=1}^{M} \int \ldots \int_{D_{i}}\left(\sum_{j \neq i} P_{j} \cdot P\left(X \mid C_{j}\right)\right) d x
$$

Since each of the above integrals is positive, then the risk of $R$ is minimized if and only if the feature vector $X$ is assigned to such a class $C_{k(x)}$ that (5):

$$
\delta(x)=\underset{1 \leq i \leq M}{\arg \min } \sum_{j \neq i} P_{j} \cdot P\left(x \mid C_{j}\right) d x
$$

which is equivalent to an expression (6):

$$
\delta(\mathrm{x})=\underset{1 \leq i \leq \mathrm{M}}{\arg \max }\left(\mathrm{P}_{\mathrm{i}} \cdot \mathrm{P}\left(\mathrm{x} \mid \mathrm{C}_{\mathrm{i}}\right) \mathrm{dx}\right)
$$

Equation (6) defines the decision rule used in each statistical classifier, which can be applied in models using probabilistic neural networks (PNN) as well as multilayer perceptrons (MLP).

If $\delta(x)$ it means operations of classification, then the classification criterion can be understood as (7):

$$
\delta(x)=C_{k} \Leftrightarrow \underset{j \neq k}{\forall P}\left(C_{k} \mid X\right)>P\left(C_{j} \mid X\right)
$$

where: $\mathrm{X}$ is the features vector, $C_{k}$ and $C_{j}$ are recognized classes $k$ and $j$, respectively.

Multilayer perceptrons are often used as classifiers because after proper training they perform approximation of the conditional probability (a'posteriori) $P\left(C_{k} \mid x\right)$ of the occurrence of a given class $C_{k}$ at the output of $y_{k}$, when the characteristic features vector $X$ is given on their input. This is the most desirable feature in the diagnosis with the random uncertainty model, because these probabilities are used in the decision rule (7). Hence, a binary classifier can easily get 
from the statistical classifier.

However, to obtain probabilities $P\left(C_{k} \mid X\right)$ from a neural network, there is a need to train this network by minimizing the mean square error for the node. One possible solution is the gradient drop method. This approach is the basis for the classical method of backward propagation of errors (EBP). There are several modifications to the learning method based on EBP. One of them is the method extended by the smoothing factor that tends to continue searching for the direction selected in the previous steps. In these methods, the weight of the neural network is changed after each teaching process according to the following rule (8-10):

$$
\begin{gathered}
\Delta w_{i j}^{t}=\eta\left[(1-\mu) \delta_{i}^{t} y_{j}^{t}+\mu \Delta w_{i j}^{t-1}\right] \\
\delta_{i_{i}}^{(t)}=\frac{\partial y_{i}^{(t)}}{\partial \operatorname{net}_{i}^{(t)}}\left(z_{i}^{(t)}-y_{i}^{(t)}\right) \\
\partial_{i}^{(t)}=\frac{\partial z_{i}^{(t)}}{\partial \operatorname{net}_{i}^{(t)}} \sum_{k=1}^{k} \delta_{k}^{(t)} w_{k i}^{(t)}
\end{gathered}
$$

where: $\Delta w_{i j}$ is the change in the weight of neurons from $\mathrm{j}$ to $\mathrm{i}$, after training the network in step $t, \eta$ is the teaching factor, $m$ is the smoothing factor, and $y_{j}{ }^{(t)}$ is the output of neuron $j$.

The meaning of $\delta_{i}^{(t)}$ in equation (8) depends on the neuron layer. If the neuron is in the output layer, the $\delta_{i}^{(t)}$ is calculated according to the relationship (9), otherwise it is calculated according to the relationship (10). In equation (9) net ${ }^{(t)}$ is the excitation of the neuron network $i, z_{i}^{(t)}$ is the expected value on neuron $i$, after undergoing training in step $t$, while $K$ is the number of neurons in the output layer.
In this work, a statistical classifier is proposed as a PNN. Such networks, which are nuclear estimators of probability density functions, are built as a special type of radial neural networks dedicated to estimation of probability density functions.

From the network architecture point of view, the PNN is a three-layer network without feedbacks (Fig. 3). It consists of an input layer, pattern layer and summation layer.

The input layer is composed of $\mathrm{N}$ elements to process $\mathrm{N}$-dimensional features vectors generated by RWD $\left(\mathrm{N}=\mathrm{N}_{\mathrm{R}}+\mathrm{N}_{\mathrm{W}}\right)$.

The pattern layer consists of $M$ groups of pattern neurons associated with $\mathrm{M}$ classes. Radial neurons with Gaussian transition function are proposed in this layer. Each pattern layer neuron is connected to each input layer neuron, and the pattern layer weight vectors are equal to the characteristic features' vectors used in the training set. Unlike the pattern layer, the summation layer consisting of $\mathrm{M}$ neurons is organized in such a way that only one output neuron is connected to neurons from any group of patterns.

\section{EXPERIMENTAL RESULTS}

The goal of the following experiment is:

- determining whether classification of fingerprints by the neural network is possible,

- determining the quality (accuracy) of classification by the optoelectronic system (RWD + ANN),

- determining the advantages of classification (recognition) using spectral power density (are these signals only from rings or only wedges or from wedges and rings (correlations of edges and their angles)), which are obtained from the extractor of characteristic features in the form of RWD.

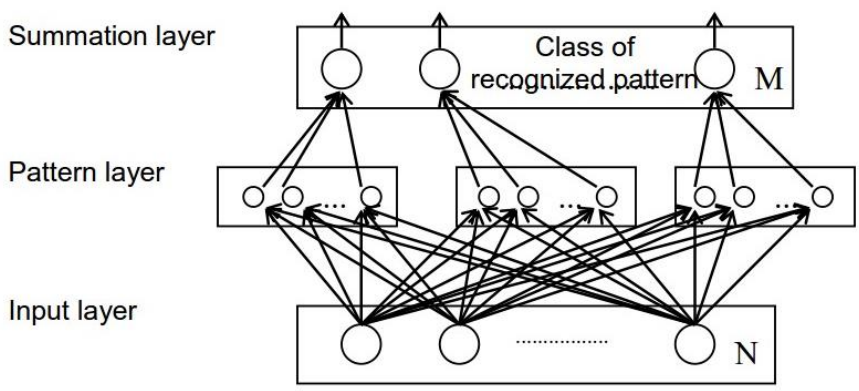

Characteristic features of patterns generated by HRWD

Fig. 3. PNN classifying the vector of characteristic features generated by the features extractor in the form of RWD 


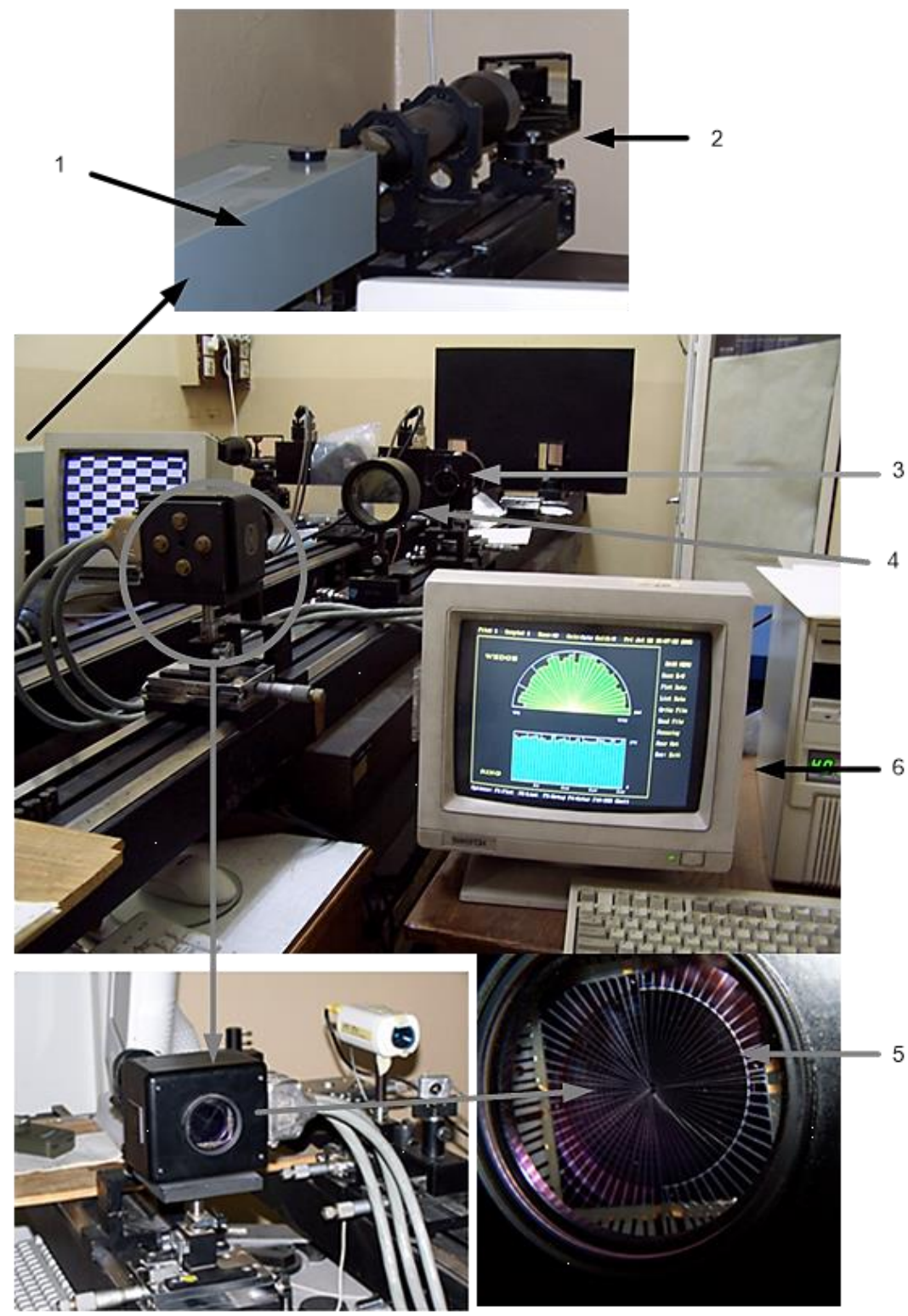

Fig. 4. Laboratory stand for images recognition in the diffraction plane, 1 - laser, 2 - collimator, 3 - spatial modulator, 4 - lens, 5 - ring-wedge detector, 6 - computer as a classifier in the artificial neural network

Classification of fingerprints is in practice a serious challenge for diffractive image sampling, since it is obvious that data from both ring and wedge are necessary for recognition. From a physical point of view, it is known that features, such as spectral power density, are important, but angles and edges are also important. Therefore, methods based on neural networks of characteristic features classification may be useful in this problem.

The proposal of the laboratory stand for the classification and identification of motor vehicle users (on the example of fingerprints) is presented in Figure 4. 
The laser light beam (Fig. 4) formed by the collimator falls on the spatial light modulator on which the recognized fingerprint image is displayed. Then the optical information is directed to the lens which implements the Fourier transform. In the focal length of the lens, in the diffraction plane, there is a feature extractor in the form of a photodetectors matrix (photodiodes). The matrix is divided into half-rings and wedges. Each wedge and half-ring is designed to sample (integrate the intensity) of incident radiation and convert it to an electric signal, which is a measure of the characteristics features of the object being tested.

The system contains two basic (main) components: the characteristics features' extractor (RWD) and the ANN. The task of RWD is to extract the vector of characteristics features from the input image. The Fourier transform process has been implemented optically by the lens, and therefore the very time-consuming operation (compared to the discrete Fourier transform) is performed immediately. RWD works fast, so processing of the input image into the vector of characteristics features is performed in near real time.

The vector of characteristics features consists only of several dozen (64) elements and the digital processing of such compressed data can be implemented extremely efficiently. Reducing the dimensions of the characteristics features' vector compared to the dimensions of the characteristics features of the input image is the most important reason for using this type of extractors.

Figure 5 shows the input data set - thumbprints taken from eight people $(\mathrm{G} 1, \mathrm{G} 2, \ldots \mathrm{G} 8)$. Thirteen $(8+5)$ fingerprints were taken and recorded, so the data could be separated into 5 learning sets and 8 test sets. The photos below show one set of different 8 tested thumb images (from $\mathrm{G} 1$ up left to G8 down right).
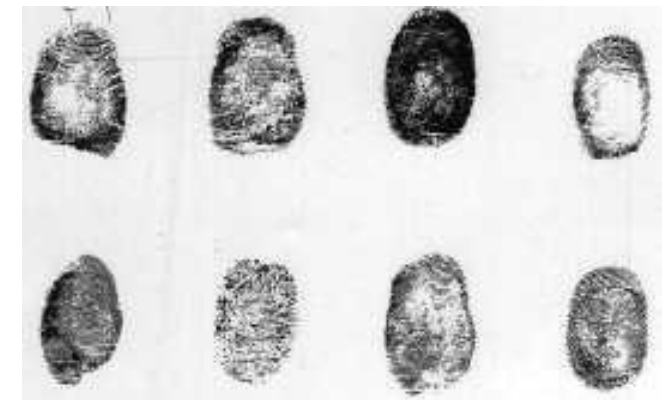

Fig. 5. Thumbprints (G1, G2, ..., G8) taken from 8 people

Obtained fingerprints (Fig. 5) usually are nonsharp. Thus, the pre-treatment problem was treated as recognition of noisy images. The wavelet method for detecting non-sharp edges with the Haar-Gauss wavelet (11) was used to preliminary preparation of images - fingerprints, which were data for controlling the spatial light modulator.

$$
h_{s}(x)=\frac{2}{s}\left(\frac{x-q}{s}\right) \cdot \exp \left[-\left(\frac{x-q}{s}\right)^{2}\right]-\frac{2}{s}\left(\frac{x+q}{s}\right) \cdot \exp \left[-\left(\frac{x+q}{s}\right)^{2}\right]
$$

Haar-Gaussian wavelet transform $W(g(v))$ of the non-sharp edge function $g(v)$ has defined as (12):

$$
W\{g(v)\}=\int_{-\infty}^{\infty} H(v) \cdot G(v) \cdot \exp (i 2 \pi v) d v
$$

where: $\mathrm{H}(v)$ and $\mathrm{G}(v)$ are Fourier transforms of the Haar-Gauss wavelet and of the non-sharp edge function $g(v)$, respectively. Therefore (13):

$$
W\{f(v)\}=\int_{-\infty}^{\infty} H_{h}(v) \cdot F(v) \cdot \exp (i 2 \pi v x) d v=\frac{2 a s}{\sqrt{s^{2}+\sigma^{2}}}\left[e^{-\left(\frac{x-x_{0}}{\sigma \mu}\right)^{2}}-e^{-\left(\frac{x+x_{0}}{\sigma \mu}\right)^{2}}\right]
$$

where: $\mu=\sqrt{1+\frac{\pi}{4 \beta^{2} q^{2}}}, x_{0}=\frac{\sqrt{\pi} \cdot \sigma}{2 \beta}$

The sharp change in the intensity of the image function (understood in this article as the edge fingerprint) is equivalent to the local maximum of the wavelet transform. The horizontal and vertical components of the gradient vector at the resolution level $j\left(d_{m, n}(j . h), d_{m, n}(j, v)\right)$ respectively are local maximum values of the wavelet transform on the horizontal and vertical axes (14). 


$$
\begin{gathered}
\left\langle f, \psi^{h}\right\rangle=d^{(j, h)}=2^{2 j} \cdot \frac{\partial}{\partial y}\left\{f(x, y) *\left[\phi_{j}(x) \cdot \Theta_{j}(y)\right]\right\} \\
\left\langle f, \psi^{v}\right\rangle=d^{(j, v)}=2^{2 j} \cdot \frac{\partial}{\partial x}\left\{f(x, y) *\left[\phi_{j}(y) \cdot \Theta_{j}(x)\right]\right\} \\
\left\langle f, \psi^{d}\right\rangle=d_{m, n}^{(j, h)}=2^{2 j} \cdot \frac{\partial^{2}}{\partial x \partial y}\left\{f(x, y) *\left[\Theta_{j}(x) \cdot \Theta_{j}(y)\right]\right\}
\end{gathered}
$$

The wavelet transformation module has been defined as (15):

$$
\left|M_{j}(x, y)\right|=\sqrt{\left|d^{(j, h)}(x, y)\right|^{2}+\left|d^{(j, v)}(x, y)\right|^{2}}
$$

It follows from the above that the gradient module is proportional to the wavelet transform module.

The results in the tables form of tests from the laboratory stand (RWD and ANN) were obtained at the Institute of Physics of the Military University of Technology realizing fingerprint recognition using information from rings, wedges and also rings and wedges of RWD. An example vector of characteristic features for the G1 thumbprint is shown in Figure 6.

Fig. 7 shows the variances of the characteristic feature from the RWD for the G1 thumbprint.

Table 1 presents the classification results of the fingerprints set (160 - obtained from 8 people (G1 to G8), where are 20 recognized thumbprints and 5 learning fingerprints) by the optoelectronic system RWD+ANN. Amplitude of signals' logarithm of the RWD (LOG (6 to 32)) obtained only from rings with large numbers (6 to 32 ) was classified.

Table 1. The results of classification of the fingerprints set with an amplitude of signals' logarithm of the RWD obtained only rings

\begin{tabular}{|c|c|c|c|c|c|c|c|c|}
\cline { 2 - 10 } \multicolumn{1}{c|}{} & \multicolumn{7}{c|}{ Output neuron } \\
\hline Test set & 1 & 2 & 3 & 4 & 5 & 6 & 7 & 8 \\
\hline G1 & 20 & & & & & & & \\
\hline G2 & & 20 & & & & & & \\
\hline G3 & & & 20 & & & & & \\
\hline G4 & & & & 20 & & & & \\
\hline G5 & & & & & 20 & & & \\
\hline G6 & & & & & & 20 & & \\
\hline G7 & & & & & $\mathbf{2}$ & & $\mathbf{1 8}$ & \\
\hline G8 & & & & & & & & 20 \\
\hline
\end{tabular}
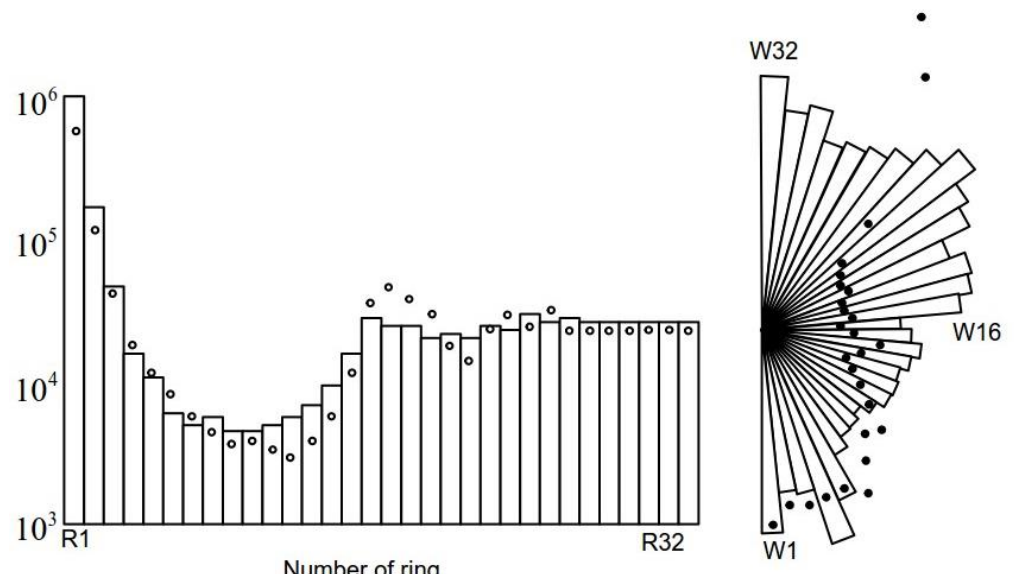

Fig. 6. The vector of characteristic features for the $\mathbf{G 1}$ thumbprint 


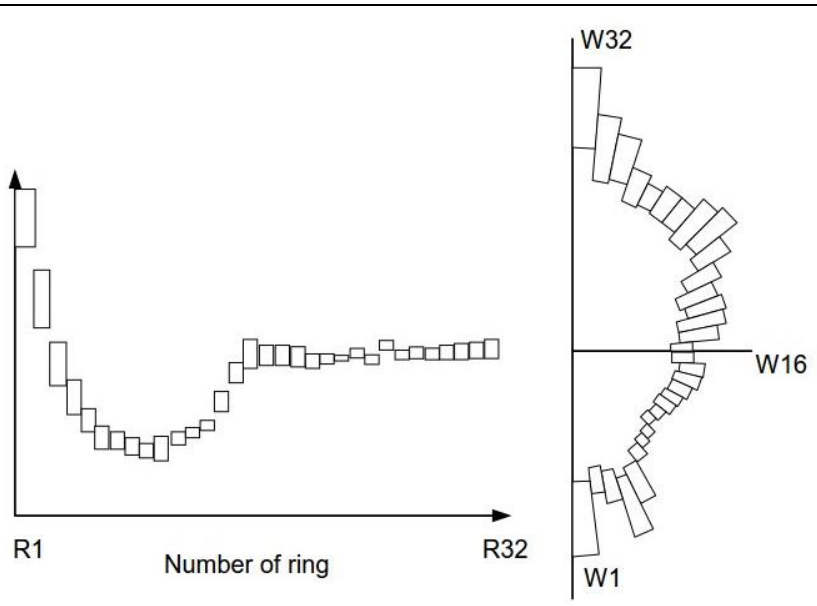

Fig. 7. Variation of the characteristic feature from the RWD for the $\mathbf{G} 1$ thumbprint

Optoelectronic system error RWD+ANN in the case of using signals only from the rings of characteristic features' extractor RWD is $2 / 160$ for a set of 160 fingerprints ( 8 people $\times 20$ fingerprints $=160$ fingerprints).

Table 2 presents the results of fingerprint classification by the optoelectronic system RWD+ANN for a set of 8 people (G1 to G8), where are 20 recognized fingerprints and 5 learning fingerprints. The amplitude of the signal's logarithm obtained only from RWD wedges was classified.

Table 2. The result of fingerprint classification with amplitude of the signal's logarithm obtained only from RWD wedges

\begin{tabular}{|c|c|c|c|c|c|c|c|c|}
\cline { 2 - 10 } \multicolumn{1}{c|}{} & \multicolumn{7}{c|}{ Output neuron } \\
\hline Test set & 1 & 2 & 3 & 4 & 5 & 6 & $\mathbf{7}$ & 8 \\
\hline G1 & 20 & & & & & & & \\
\hline G2 & & $\mathbf{1 8}$ & & & & $\mathbf{2}$ & & \\
\hline G3 & & & $\mathbf{1 8}$ & & & & $\mathbf{2}$ & \\
\hline G4 & & & & 20 & & & & \\
\hline G5 & & & & & 20 & & & \\
\hline G6 & & & & & & $\mathbf{1 8}$ & & $\mathbf{2}$ \\
\hline G7 & & & 1 & & & & $\mathbf{1 9}$ & \\
\hline G8 & & & & & & & & 20 \\
\hline
\end{tabular}

The error level of the optoelectronic system (case - only signals from RWD wedges, and 7 fingerprints were incorrectly classified from 160 fingerprints) is $7 / 160$.

Table 3 presents the results of fingerprint classification by the optoelectronic system RWD+ANN for a set of 8 people (G1 to G8), where are 20 recognized fingerprints and 5 learning fingerprints. The amplitude of the signal's logarithm from the RWD obtained from rings with large numbers and from RWD wedges was classified.

Table 3. The results of fingerprint classification with amplitude of the signal's logarithm from the RWD obtained from rings with large numbers and from RWD wedges

\begin{tabular}{|c|c|c|c|c|c|c|c|c|}
\cline { 2 - 9 } \multicolumn{1}{c|}{} & \multicolumn{7}{c|}{ Output neuron } \\
\hline Test set & 1 & 2 & 3 & 4 & 5 & 6 & 7 & 8 \\
\hline G1 & 20 & & & & & & & \\
\hline G2 & & 20 & & & & & & \\
\hline G3 & & & 20 & & & & & \\
\hline G4 & & & & 20 & & & & \\
\hline G5 & & & & & 20 & & & \\
\hline G6 & & & & & & 20 & & \\
\hline G7 & & & & & 1 & & 19 & \\
\hline G8 & & & & & & & & 20 \\
\hline
\end{tabular}

Optoelectronic system error using rings and wedges RWD+ANN (case) is 1/160.

Table 4 summarizes the classification possibilities of fingerprints by the laboratory optoelectronic system RWD+ANN.

Table 4. The classification possibilities of fingerprints

\begin{tabular}{|c|c|c|c|c|c|c|c|c|}
\cline { 2 - 9 } \multicolumn{1}{c|}{} & \multicolumn{7}{c|}{ Output neuron } \\
\hline Test set & 1 & 2 & 3 & 4 & 5 & 6 & 7 & 8 \\
\hline G1 & & & & & & & & \\
\hline G2 & & & & & & $\Delta \mathbf{\Delta}$ & & \\
\hline G3 & & & & & & & $\Delta \Delta$ & \\
\hline G4 & & & & & & & & \\
\hline G5 & & & & & & & & $\Delta \Delta$ \\
\hline G6 & & & & $\Delta^{*}$ & & & & \\
\hline G7 & & & $\Delta$ & & O O & & & \\
\hline G8 & & & & & & & & \\
\hline
\end{tabular}

Below there are the results of fingerprint classification by the laboratory optoelectronic 
system RWD + ANN in the form of an error level for a set of 160 fingerprints for the accepted following conditions:

\begin{tabular}{|l|c|c|}
\hline wedge+ring & $\left(^{*}\right)$ & $1 / 160$ \\
\hline ring & $(\mathrm{O})$ & $2 / 160$ \\
\hline wedge & $(\Delta)$ & $7 / 160$ \\
\hline
\end{tabular}

The conclusion is that the smallest system classification error is $1 / 160$, while the largest is $7 / 160$.

\section{ConClusions}

A simple optoelectronic (hybrid) system for image recognition (fingerprints) with complex internal texture has been proposed. The system can be successfully used to recognize the images of face, handwriting or license plates of motor vehicles. The system can also be successfully used to identify material defects. Extraction of the characteristic features' vector is carried out in close to real time. The system can be easily implemented in the software version. The conducted experiments showed the high effectiveness of the proposed optoelectronic system.

\section{ABBREVIATIONS}

1. ANN - Artificial Neural Network;

2. ATR - Automatic Target Recognition;

3. EBP - Error Back Propagation;

4. MLP-Multilayer Perceptrons;

5. PNN - Probabilistic Neural Network;

6. RWD-Ring-Wedge Detector.

\section{METODA AUTOMATYCZNEJ IDENTYFIKACJ UŻYTKOWNIKÓW POJAZDÓW MECHANICZNYCH}

Praca prezentuje metodę automatycznego rozpoznawania obrazów dyfrakcyjnych odcisków palców użytkowników pojazdów mechanicznych. Proponowana metoda, bazuje na podstawowych właściwościach fizycznych transformaty Fouriera. Stwarza możliwość sprowadzenia problemu rozpoznawania do transformaty Fouriera funkcji obrazowej, ekstrakcji wektora cech charakterystycznych i klasyfikacji obrazów wejściowych.

Słowa kluczowe: sztuczna sieć neuronowa, automatyczna identyfikacja, wektor cech, układ optoelektroniczny, detektor pierścieniowo-klinowy

\section{REFERENCES}

[1] Casasent D., Song J. (1985) “A Computer Generated Hologram for Diffraction-Pattern Sampling". Proc. SPIE. 0523, Applications of Holography, pp 227-236. https://doi.org/10.1117/12.946287

[2] George N., Wang S., Venable D. L. (1989) “Pattern recognition using the ring-wedge detector and neural network software". Proceedings of SPIE: Optical Pattern Recognition II, Vol. 1134, pp 96-106. https://doi.org/10.1117/12.961621

[3] George N., Wang S. (1994) "Neural Networks Applied to Diffraction-Pattern Sampling". Applied Optics. Vol. 33, Issue 14, pp 3127-3134.

https://doi.org/10.1364/AO.33.003127

[4] Raghu P. P., Yegnanrayana B. (1998) "Supervised Texture Classification using a probabilistic Neural Network and Constraint Satisfaction Model". IEEE Trans. Neural Networks, 9 (3), pp 516-522.

DOl: $10.1109 / 72.668893$

[5] Niedziela T.(2000) “Optoelektroniczne metody rozpoznawania obrazów". Prace Naukowe Instytutu Technicznego Wojsk Lotniczych. No. 9, ISSN 1234-3544, pp 93-105

[6] Cyran K. A., Jaroszewicz L. R., Niedziela T. (2001) “Neural Network based Automatic Diffraction Pattern Recognition". Opto-electronics Review. Vol. 9, No. 3, ISSN 1230-3402, pp 301-307

[7] Cyran K. A., Jaroszewicz L. R., Niedziela T., Merta I. (2001) "Concurrent signal processing in optimized hybrid CGH-ANN systems". Optica Applicata, XXXI, 4, ISSN 0078-5466, pp 675-689

[8] Ganotra D., Joseph K., Singh J. (2002) "Neural network based face recognition by using diffraction pattern sampling with a digital ring-wedge detector". Optics Communications, Vol. 202, pp 61-68

https://doi.org/10.1016/S0030-4018(02)01088-X

[9] Ganotra D., Joseph K., Singh J. (2004) "Modified geometry of ring-wedge detector for sampling Fourier transform of fingerprints for classification using neural networks", Optics and Lasers in Engineering, Vol. 42, pp 167-177. https://doi.org/10.1016/j.optlaseng.2003.08.003

[10] Rusyn B., Prudyus I., Ostap V. (2001) "Fingerprint Image Enhancement Algorithm". The Experience of Designing and Application of CAD Systems in Microelectronics, Proceedings of the $6^{\text {th }}$ International Conference CADSM 2001, pp 193-194. DOI: 10.1109/CADSM.2001.975804

[11] Varetskyy Y., Rusyn B., Molga A., Ignatovych A. (2010) "ANew Method of Fingerprint Key Protection of Grid Credential". In: Choraś R.S. (eds) Image Processing and Communications Challenges 2. Advances in Intelligent and Soft Computing, Vol. 84, Springer-Verlag, Berlin, Heidelberg, ISBN 978-3-64216294-7, pp 99-103.

https://doi.org/10.1007/978-3-642-16295-4_11 